\title{
Clinical and radiobiological consideration of cyclical hypofractionated radiation therapy also known as QUAD Shot for neglected skin cancer disfiguring the face of a non- compliant patient who was refusing surgery and protracted radiation therapy: case report
}

\author{
Whoon Jong Kil', Kevin Camphausen ${ }^{2}$, In Hye $\mathrm{Cho}^{3}$ \\ ${ }^{1}$ Radiation Oncology, WellSpan Health, Chambersburg, PA, USA \\ ${ }^{2}$ Radiation Oncology Branch, National Cancer Institute, National Institute of Health, Bethesda, MD, USA \\ ${ }^{3}$ Independent Research, Washington, DC, USA
}

\begin{abstract}
Although surgery is the mainstay of local treatment for skin cancer, definitive radiation therapy (RT) has been also applied for patients who are unable to tolerate surgery. Definitive RT regimens usually consist of daily treatment for 4-7 weeks. Such protracted daily RT regimens, however, would not be feasible for non-compliant patients or patients who are unable to make multiple daily trips for weeks. Without treatment, however, skin cancers can continuously progress and cause distressing symptoms. A cyclical hypofractionated RT (QUAD Shot: 14 Gy in 4 fractions, twice-daily treatments with 6 hours interval on 2 consecutive days) can be a practical RT regimen for those patients. In this report, we present the successful treatment course of repeated QUAD Shots in a 79-year-old patient with neglected skin cancer that was disfiguring his face yet declined definitive surgery and protracted RT. We also evaluated and compared biologically equivalent doses between QUAD Shots and conventionally fractionated protracted RT regimens.
\end{abstract}

Keywords: Neglected skin cancer, Non-compliant, Disfiguring face, QUAD Shots, BED

\section{Introduction}

Non-melanoma skin cancers are the most common cancers worldwide with cutaneous squamous cell carcinoma (CSCC) accounting for the second largest percentage next to basal cell carcinoma $[1,2]$. The risk of CSCC increases with age and in sun-exposed areas [3-5]. Recent studies show that the incidence of CSCC has been rising more rapidly than basal cell carcinoma [2]. Localized cSCC has an excellent prognosis with 5 -year survival of $\geq 90 \%$ after treatments $[2,6,7]$. However, neglected localized early stage cSCC without treatment can progress to neighboring organs causing substantial local area destruction, disfigurement, and functional loss. Patients with elderly age, social isolation, multiple co-morbidities, or non-compliance to medical advice are at high risk of neglecting early stage cSCC. Treatment for neglected CSCC is

Received 13 May 2019, Revised 31 May 2019, Accepted 04 June 2019.

Correspondence: Whoon Jong Kil, Radiation Oncology, WellSpan Health, 260 N 7th St., Chambersburg, PA, USA. Tel: +1717-262-4660, Fax: +1-717-263-6251, E-mail: whoonkil@gmail.com (http://orcid.org/0000-0001-7631-2949)

(c) This is an Open Access article distributed under the terms of the Creative Commons Attribution Non-Commercial License (http://creativecommons.org/ licenses/by-nc/4.0/) which permits unrestricted non-commercial use, distribution, and reproduction in any medium, provided the original work is properly cited.

www.e-roj.org 

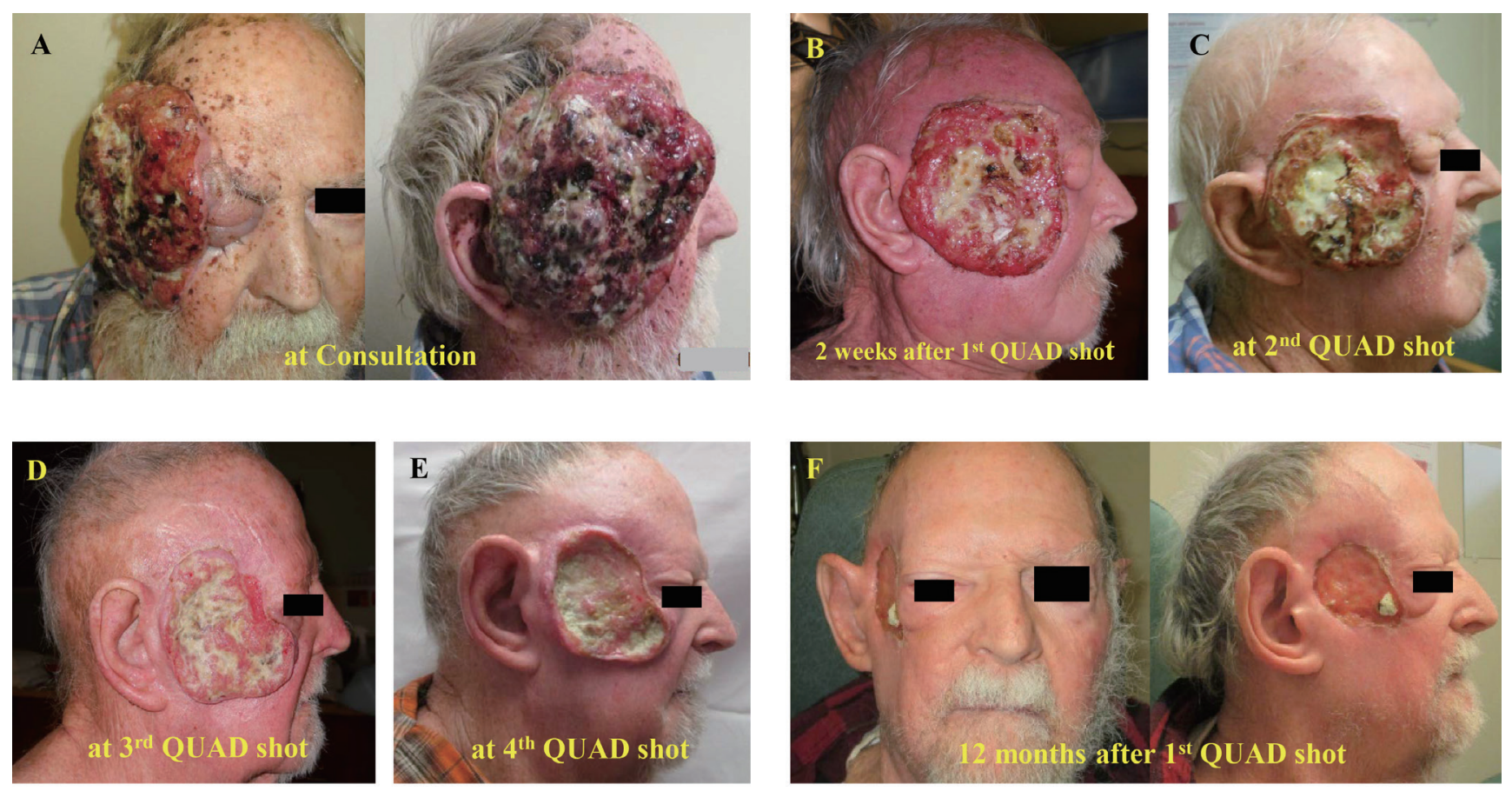

Fig. 1. Face pictures of a patient with neglected extensive cutaneous squamous cell carcinoma before cyclical hypofractionated radiotherapy (QUAD Shot) (A); at 2 weeks after the first QUAD Shot (B); at the second (C), third (D) and fourth QUAD Shot (E); and at 12 months after the first QUAD Shot (F).

often complicated, requiring extensive surgeries followed by adjuvant treatments.

Although surgery is the mainstay of local treatment for skin cancer, radiotherapy (RT) has been used as the primary treatment in cases where surgery would cause significant cosmetic and/or functional deficits or depending on patient preference of local therapy $[8,9]$. Definitive RT regimens for skin cancers usually consist of daily treatments for weeks [10]. However, protracted daily RT regimens would not be feasible for non-compliant patients or patients who are unable to make multiple daily trips for weeks due to social or physical limitations. Without local treatment, however, neglected cSCC can continuously progress and cause distressing symptoms, such as functional impairment, severe pain, and cancer-related wounds, bleeding, and infection.

Herein, we present the clinical course of repeated cyclical hypofractionated RT (also known as QUAD Shot) in a 79-yearold patient with neglected CSCC that was disfiguring his face yet declined definitive surgery and multiple weeks of daily RT. We also evaluated and compared biologically equivalent doses (BEDs) between QUAD Shots and conventionally fractionated protracted RT regimens.

\section{Case Report}

A 78-year-old socially isolated male with history of noncompliance to medical advice presented in a hospital after recent multiple falls at home. At the hospital, he was found to have a huge, ulcerative and bleeding mass disfiguring the right side of his face (Fig. 1A). The patient stated that he had the mass on the right side of his face for many years, had a biopsy on the mass showing CSCC three years ago at the other medical facility but declined surgery or any treatments at that time (data was not available). Subsequently, a surgery consultation was made for biopsy and to get a surgical opinion to manage this neglected facial lesion. Biopsy confirmed cSCC. Computed tomography (CT) scan showed an $11 \times 10 \times 3 \mathrm{~cm}$ soft tissue mass arising from the right side of his face and temporal area. The mass invaded the myofascial plane of the right temporalis muscle, but not the facial bone (Fig. 2A). There were several enlarged right submandibular and upper cervical neck lymph nodes. Positron emission tomography (PET) scan demonstrated hypermetabolic masses involving the right face and frontotemporal scalp with maximum standardized uptake values (SUVs) of 18 (Fig. 2B) and right neck level I-II lymph nodal station with maximum SUVs of 4.9 (Fig. 2C). The patient again declined to receive extensive surgeries for this neglected 

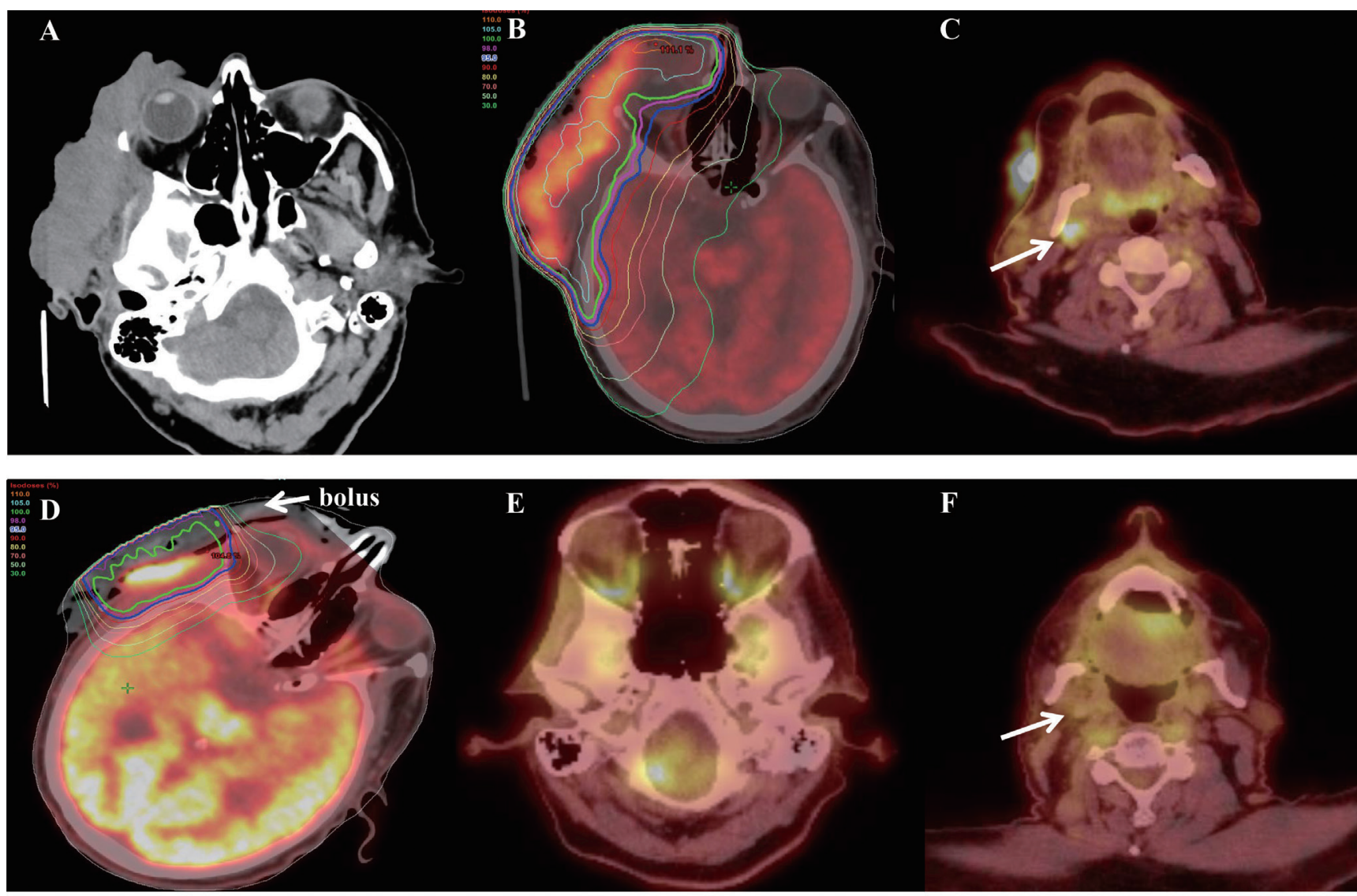

Fig. 2. Computed tomography (CT) before cyclical hypofractionated radiotherapy (QUAD Shot) (A), positron emission tomography-CT at the first $(B, C)$, fourth QUAD Shot $(D)$ and at 12 months after the first QUAD Shot $(E, F)$.

CSCC on the right face. Then, he was referred for RT.

During the radiation oncology consultation, a huge mass on the right side of his face with a foul-odor, necrotic and bleeding surface extending into the right-side eyelid, ear lobe and temporal scalp was noticed. He also presented weakness and trismus in the right face (Fig. 1A). Motion and sensation in the left face were grossly intact. There was no palpable Iymphadenopathy in the neck. He refused to make weeks of daily trips for definitive RT but would consider less than two trips. Thus, a radiation oncologist suggested cyclical hypofractionated RT also known as QUAD Shot to CSCC on the face and hypermetabolic nodes in the neck for local disease control and symptom palliation. To receive a QUAD Shot, the patient would need to visit the radiation oncology clinic for 2 consecutive days, which could be repeated every 3-4 weeks for at least three times depending on the tumor response and patient's tolerability. Patient agreed to try the first QUAD Shot and wanted to decide further treatments thereafter.

The first QUAD Shot consisted of $14 \mathrm{~Gy}$ in 4 fractions, given twice a day, 6 hours apart, for 2 consecutive days, to hypermetabolic gross diseases in the right face and neck including right eyelid, right parotid gland, involved right masticate space, and right trigeminal nerve and facial nerve tracts to the skull base using an intensity-modulated RT. A 1.0$\mathrm{cm}$ bolus was applied over CSCC on the right face to ensure the surface of the tumor would get the full prescription dose. Planning objectives required the planning target volume coverage of $95 \%$ to $110 \%$. Radiation dose to the spinal cord brain stem, and optic nerve/chiasm were limited to 9 Gy per QUAD Shot. For other organs at risk, the constraints were 'as low as reasonably achievable' (Fig. 2B).

Two weeks after the first QUAD Shot, the patient noticed his CSCC on the right face became significantly decreased in size and bled less (Fig. 1B), which motivated him to pursue additional RT. The subsequent QUAD Shots were planned on new computed tomography (CT)-simulation scans and were given to gross residual diseases. He successfully completed the second and third QUAD Shot every 3-4 weeks resulting in continuous shrinkage of $\mathrm{CSCC}$ on the face (Fig. $1 \mathrm{C}$ and 1D, respectively). Two weeks after the third QUAD Shot, he 
Table 1. BED comparison between the various RT regimens

\begin{tabular}{lccccc}
\hline RT regimen & $\begin{array}{c}\text { Overall RT time } \\
\text { (day) }\end{array}$ & BED of Gy $y_{10}$ & $\begin{array}{c}\text { Proliferation } \\
\text { correction }\end{array}$ & BED $_{\text {corrected }}$ of Gy $_{10}$ & BED of Gy $_{3}$ \\
\hline $1.8 \mathrm{~Gy} \times 25$ & 33 & 53.10 & -15.24 & 37.85 & 72.00 \\
$2.0 \mathrm{~Gy} \times 25$ & 33 & 60.00 & -15.24 & 44.75 & 83.33 \\
$2.0 \mathrm{~Gy} \times 27$ & 37 & 64.80 & -17.09 & 47.70 & 90.00 \\
$2.0 \mathrm{~Gy} \times 30$ & 40 & 72.00 & -18.47 & 53.52 & 100.00 \\
2.0 Gy $\times 35$ & 47 & 19.00 & -21.71 & 62.28 & 116.66 \\
QUAD Shot $\times 1$ & 2 & 39.46 & -0.92 & 18.80 & 32.16 \\
QUAD Shot $\times 2$ & 23 & 58.32 & -10.62 & 28.83 & 64.32 \\
QUAD Shot $\times 3$ & 44 & 78.91 & -20.32 & 37.99 & 96.48 \\
QUAD Shot $\times 4$ & 65 & 98.64 & -30.02 & 48.88 & 128.64 \\
QUAD Shot $\times 5$ & 86 & -39.72 & 58.91 & 160.80 \\
\hline
\end{tabular}

BED, biologically effective dose; RT, radiation therapy; QUAD Shot, cyclical hypofractionated RT to deliver 14-14.4 Gy in 4 fractions, given twice a day, 6 hours apart, for 2 consecutive days, repeated every 3 weeks; Overall RT time, days required to complete planned RT assuming RT begins on Monday and no treatment interruption; $\mathrm{Gy}_{10}$, calculated BED using $\alpha / \beta$ ratio to be 10 Gy for tumor or early responding tissue; $\mathrm{Gy}_{3}$, calculated $\mathrm{BED}$ using $\alpha / \beta$ ratio to be $3 \mathrm{~Gy}$ for late responding tissue; Proliferation correction was calculated with 5 days of potential doubling time and $0.3 \mathrm{~Gy}$ of ' $\alpha$ ' representing the log of the cell killed per Gy; BED $_{\text {corrected }}$ proliferation corrected BED.

returned to the radiation oncology clinic for follow-up. While there was mild ptosis, he was able to open his right eye. Vison and eyeball movements were grossly intact. Improved trismus and weakness in the right face were also observed. Patient experienced slightly decreased salivation and hair loss in the right lateral scalp, eye brow, and jaw. Although the previous three cycles of QUAD (OUAD Shot $\times 3$ ) Shots substantially decreased the neglected CSCC on the right side of his face, there was still residual painful induration with focal necrosis and foul-odor around the posterior and inferior parts of the tumor bed (Fig. 1E). An additional QUAD Shot was requested by the patient to alleviate the residual painful induration in the tumor bed.

Before considering further local treatment, he underwent repeat PET-CT scan revealing a significant decrease in size and metabolic activity (SUV of 4) in the right facial tumor (Fig. 2D), no metabolic changes in the right neck lymph node and no disease elsewhere. Subsequently, a surgical option for the residual disease was sought. However, surgeons considered him to be unfit for extensive surgery, then recommended him for further local RT if possible. After discussions regarding the risks and benefits from additional $\mathrm{RT}$, he elected to pursue the fourth QUAD Shot.

The fourth QUAD Shot was planned on new CT-simulation scan with $9 \mathrm{MeV}$-electron with $1.0 \mathrm{~cm}$ bolus covering the surface of the tumor bed (Fig. 2D). The accumulated doses to the optic nerve, optic chiasm, brain, and spinal cord were less than 36 Gy from all QUAD $\times 4$. He successfully completed the fourth QUAD Shot. One month after the last QUAD Shot, he reported no pain or foul-odor in the tumor bed, which positively affected on his quality of life and motivated him to pursue a palliative maintenance immunotherapy (cemiplimab at a medical oncologist's discretion). On his last clinic visit 12 months after the first QUAD Shot, the patient demonstrated a restored face with minimum facial weakness and regenerating skin around the tumor bed (Fig. 1F) without local disease progression in repeated PET-CT scan (Fig. 2E, 2F).

Institutional Review Board waived a board review on the case report. The patient has given signed consent for reporting this report.

\section{Discussion and Conclusion}

Although definitive RT for skin cancers has shown excellent local tumor control and cosmetic outcome (particularly in facial lesions), protracted daily RT regimens can be a huge challenge for patients with lack of social-economic support, physical impairment, or non-compliance to daily treatments. Short-course palliative daily RT regimens (20-30 Gy in 5-10 fractions) for cancer in the head and neck region demonstrated only modest rate of symptom palliation (50\%-60\%) with high acute grade 3 or higher (grade 3+) mucositis (62\%) and dermatitis (56\%) $[11,12]$. Among RT regimens, a QUAD Shot requires only 2 consecutive days of hospital visits per each cycle yet has shown excellent local tumor control (53\%-77\%) and symptom palliation (56\%-85\%) with minimum side 
Table 2. BED to the organs at risk from equal or more than QUAD Shot $\times 3$

\begin{tabular}{|c|c|c|c|c|c|c|}
\hline \multirow{2}{*}{$\begin{array}{c}\text { Dose per QUAD } \\
\text { Shot }\end{array}$} & \multicolumn{3}{|c|}{ BED $_{\text {corrected }}$ of $\mathrm{Gy}_{10}$ from QUAD Shot } & \multicolumn{3}{|c|}{ BED of $\mathrm{Gy}_{3}$ from QUAD Shot } \\
\hline & 3 cycles & 4 cycles & 5 cycles & 3 cycles & 4 cycles & 5 cycles \\
\hline $13 \mathrm{~Gy}$ & 31.35 & 38.87 & 46.40 & 81.25 & 108.33 & 135.42 \\
\hline $12 \mathrm{~Gy}$ & 26.47 & 32.37 & 38.27 & 72.00 & 96.00 & 120.00 \\
\hline $11 \mathrm{~Gy}$ & 21.75 & 26.08 & 30.40 & 63.25 & 84.33 & 105.42 \\
\hline $10 \mathrm{~Gy}$ & 17.17 & 19.97 & 22.77 & 55.00 & 73.33 & 91.67 \\
\hline 9 Gy & 12.75 & 14.07 & 15.40 & 47.25 & 63.00 & 78.75 \\
\hline 8 Gy & 8.47 & 8.37 & 8.27 & 40.00 & 53.33 & 66.67 \\
\hline
\end{tabular}

BED, biologically effective dose; RT, radiation therapy; QUAD Shot, cyclical hypofractionated RT to deliver 14-14.4 Gy in 4 fractions, given twice a day, 6 hours apart, for 2 consecutive days, repeated every 3 weeks; Overall RT time, days required to complete planned RT assuming RT begins on Monday and no treatment interruption; $\mathrm{Gy}_{10}$, calculated BED using $\alpha / \beta$ ratio to be 10 Gy for tumor or early responding tissue; $\mathrm{Gy}_{3}$, calculated $\mathrm{BED}$ using $\alpha / \beta$ ratio to be $3 \mathrm{~Gy}$ for late responding tissue; Proliferation correction was calculated with 5 days of potential doubling time and $0.3 \mathrm{~Gy}$ of ' $\alpha$ ' representing the log of the cell killed per $\mathrm{Gy}$; $\mathrm{BED}_{\text {corrected }}$ proliferation corrected BED.

effects (grade $3+, 6 \%-9 \%)$ in cancer patients [11-15].

Table 1 shows the effect of overall RT times and tumor proliferation on the BED characteristic of the various RT regimens. For the BED calculation, the $\alpha / \beta$ ratio of $3 \mathrm{~Gy}$ and 10 $\mathrm{Gy}$ is used for late-responding tissues $\left(\mathrm{Gy}_{3}\right)$ and tumor or earlyresponding tissues $\left(\mathrm{Gy}_{10}\right)$. To account for tumor proliferation during the entire course of RT, a median value of 5 days for the potential doubling time and 0.3 for ' $\alpha$ ' representing the loge of the cells killed per Gy was applied in the BED calculation as previously reported [11]. Whereas BED from a QUAD Shot seemed relatively lower than those from other protracted RT regimens, repeated QUAD Shots had shown excellent tumor responses: 100\% with QUAD Shot $\times 3 ; 44 \%-50 \%$ with less than QUAD shot $x 3$ [13]. Furthermore, QUAD Shot $\times 3$ resulted in 31\%-47\% of complete tumor response rate despite lower proliferation corrected BED (BED corrected $\left._{\text {of }} 37.99 \mathrm{~Gy}_{10}\right)$ compared to definitive RT regimens (53.52-62.28 Gy $\mathrm{G}_{10}$ in $2 \mathrm{~Gy} \times 30-35$ fractions) $[13,14]$. Interestingly, the calculated $B E D_{\text {corrected }}$ of $\mathrm{Gy}_{10}$ from four and five cycles of QUAD Shot (48.88 and 58.91 $\mathrm{Gy}_{10}$, respectively) are comparable to those from protracted definitive RT regimens. Thus, as long as the organs at risk (OARs) would tolerate it, additional QUAD Shot(s) may be considered for selected patients with limited residual disease after three cycles of QUAD Shots. With advanced technology (such as an intensity-modulated RT or proton RT), highly conformal QUAD Shots can permit a higher radiation dose to the tumor while sparing the OARs compared to the two- or three-dimensional RT techniques. However, there has been limited information regarding radiation dose constraints to the OARs during three or more cycles of QUAD Shots.

To suggest radiation dose constrains to the OARs during QUAD Shot, BEDs from various radiation doses per QUAD Shot
(Gy/OUAD) in equal or more than QUAD Shot $\times 3$ regimens are calculated and listed in Table 2. For example, limiting dose to 13 Gy/QUAD in QUAD Shot $\times 4$ and 5 regimen appeared be appropriate since its $\mathrm{BED}_{\text {corrected }}$ of $\mathrm{Gy}_{10}$ are comparable to one from 45-54 Gy in 25-27 fractions (38.87-46. 40 $\mathrm{Gy}_{10}$ vs. 37.85-47.70 $\mathrm{Gy}_{10}$, respectively) for early responding tissues (such as gastrointestinal organs). For late responding tissues, Dmax to both spinal cord and optic nerve/chiasm of 13 Gy, 10 Gy and 9 Gy/QUAD in QUAD Shot $\times 3$, 4, and 5 regimens would result in $\mathrm{BED}$ of $73.33-81.25 \mathrm{~Gy}_{3}$, which are similar to those in conventionally fractionated RT regimens of 45-50 Gy in 25 fractions (72.00-83.33 Gy 3 ). For example, limiting dose to $13 \mathrm{~Gy} /$ QUAD in QUAD shot $x 4$ and 5 regimen appeared to be appropriate for early responding tissues such as gastrointestinal organs since its $\mathrm{BED}_{\text {corrected }}$ of $\mathrm{Gy}_{10}$ are comparable to one from 45-54 Gy in 25-27 fractions (38.87$46.40 \mathrm{~Gy}_{10}$ vs. $37.85-47.70 \mathrm{~Gy}_{10}$, respectively).

The main advantages of QUAD Shot regimen include high tolerance to RT with low toxicity, shortened treatment time, and excellent local tumor/symptom control compared to other protracted palliative RT regimens [11-15]. Practically QUAD Shot regimen allows planning target volume revision for each subsequent cycle of QUAD Shot to account for tumor response from previous QUAD Shot(s), which can permit less radiation dose to the OARs resulting in further lowering toxicity. The flexibility of QUAD Shot treatment based on the patient's general condition and tumor response is also one of the important clinical consideration. Certain patients with tumor progression or general medical deterioration due to other comorbidities rather than RT-related toxicity are not recommended to receive additional cycle of QUAD Shot, which would allow avoiding inefficient treatment or unnecessary 
treatment-related side effects. Although, even less than QUAD Shot $\times 3$ regimen can still offer modest rate of symptom palliation (55\%-67\%), better symptom palliation (nearly $89 \%)$ was reported in patients who received $\geq$ QUAD Shot $\times 3$ regimen $[12,13,15]$.

Whereas, within authors' knowledge, this is the first report describing radiation dose constraints to the OARs in $\geq$ QUAD Shot $\times 3$ regimens, calculated BEDs in this report should be used as a guide rather than an absolute indicator. Careful consideration of the relevant physical dose variations, the possible range of radiobiological parameters, and the individual clinical factors are also important. Seeking advice from experts in radiobiology is strongly recommended in patient-specific, different clinical situations. Further study with long-term follow-up in large numbers of selected patients treated with QUAD Shots is needed for developing new dose constraints to OARs in $\geq$ QUAD Shot $\times 3$ regimens.

In conclusion, for patients with neglected, extensive cSCC who are unable to make multiple weeks of daily trips to the clinic, QUAD Shots would be a practical RT regimen for local tumor control. Suggested BED to the OARs in this report can be used as a guide for additional QUAD Shot(s) for local tumor control in selected patients with residual CSCC after three cycles of QUAD Shots.

\section{Conflict of Interest}

No potential conflict of interest relevant to this article was reported.

\section{References}

1. Stern RS. Prevalence of a history of skin cancer in 2007: results of an incidence-based model. Arch Dermatol 2010;146:27982.

2. Rubio-Casadevall J, Hernandez-Pujol AM, Ferreira-Santos $M C$, et al. Trends in incidence and survival analysis in nonmelanoma skin cancer from 1994 to 2012 in Girona, Spain: a population-based study. Cancer Epidemiol 2016;45:6-10.

3. Xiang $F_{1}$ Lucas $R_{1}$ Hales $S$, Neale $R$. Incidence of nonmelanoma skin cancer in relation to ambient UV radiation in white populations, 1978-2012: empirical relationships. JAMA Dermatol 2014;150:1063-71.

4. Wassberg $C_{\text {, Thorn M }}$, Johansson AM, Bergstrom R, Berne
B, Ringborg U. Increasing incidence rates of squamous cell carcinoma of the skin in Sweden. Acta Derm Venereol 2001;81:268-72.

5. Hemminki $K$, Zhang $H$, Czene K. Time trends and familial risks in squamous cell carcinoma of the skin. Arch Dermatol 2003;139:885-9.

6. Eisemann $N$, Jansen $L$, Castro FA, et al. Survival with nonmelanoma skin cancer in Germany. Br J Dermatol 2016;174:778-85.

7. Schmults CD, Karia PS, Carter JB, Han J, Qureshi AA. Factors predictive of recurrence and death from cutaneous squamous cell carcinoma: a 10-year, single-institution cohort study. JAMA Dermatol 2013;149:541-7.

8. Hernandez-Machin B, Borrego L, Gil-Garcia M, Hernandez BH. Office-based radiation therapy for cutaneous carcinoma: evaluation of 710 treatments. Int J Dermatol 2007;46:453-9.

9. Grossi Marconi D, da Costa Resende B, Rauber E, et al. Head and neck non-melanoma skin cancer treated by superficial X-ray therapy: an analysis of 1021 cases. PLoS One 2016;11:e0156544.

10. National Comprehensive Cancer Network. NCCN Guidelines: squamous cell skin cancer [Internet]. Plymouth Meeting, PA: National Comprehensive Cancer Network; c2019 [cited 2019 May 30]. Available from: https://www.ncen.org/professionals/ physician_gls/pdf/squamous.pdf.

11. Kil WJ, Camphausen K. Cyclical hypofractionated radiotherapy also known as "OUAD Shot" alone using intensity-modulated radiotherapy for squamous cell carcinoma of the parotid gland in an 85-year-old patient with multiple comorbidities. Head Neck 2017;39:E55-E60.

12. Chen AM, Vaughan A, Narayan S, Vijayakumar S. Palliative radiation therapy for head and neck cancer: toward an optimal fractionation scheme. Head Neck 2008;30:1586-91.

13. Paris KJ, Spanos WJ Jr, Lindberg RD, Jose B, Albrink F. Phase I-II study of multiple daily fractions for palliation of advanced head and neck malignancies. Int J Radiat Oncol Biol Phys 1993;25:657-60.

14. Corry J, Peters $\sqcup$, Costa ID, et al. The 'QUAD SHOT': a phase II study of palliative radiotherapy for incurable head and neck cancer. Radiother Oncol 2005;77:137-42.

15. Lok BH, Jiang G, Gutiontov S, et al. Palliative head and neck radiotherapy with the RTOG 8502 regimen for incurable primary or metastatic cancers. Oral Oncol 2015;51:957-62. 